
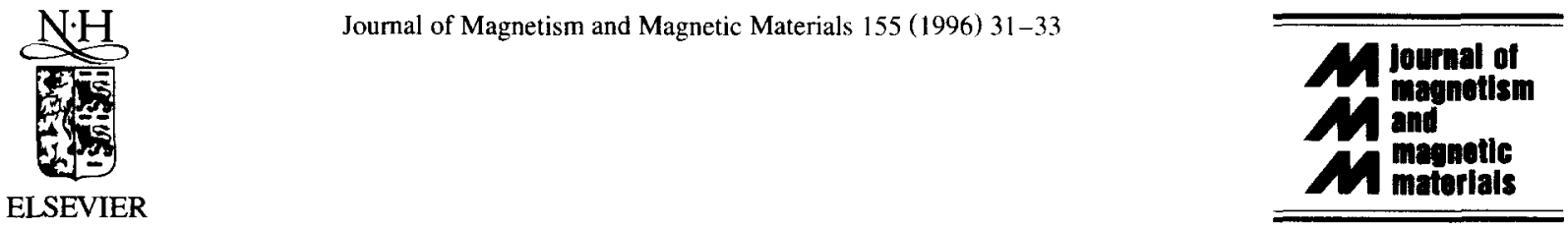

\title{
Hysteresis of hexagonal lattice arrays
}

\author{
Ivo Klik * , Ching-Ray Chang \\ Department of Physics, National Taiwan Unicersit, Taipei, Taiwan, ROC
}

\begin{abstract}
Thermal relaxation is studied in an array of interacting single domain uniaxial particles deposited on a planar hexagonal lattice. Coherent rotation of magnetization and perfect alignment of easy axes with applied field are assumed. The lattice is stretched in the direction of the easy axes and the coercivity and switching field distribution (SFD) are studied as functions of the stretching factor. Coercivity is found to have monotonic dependence on the stretching factor while the SFD function exhibits complicated behavior associated with a transition between configurations dominated by ferromagnetic and antiferromagnetic ordering.
\end{abstract}

In a recent work [1] we studied thermal relaxation in arrays of interacting single domain ferromagnetic particles and expressed the evolution of the system in terms of the occupation probabilities $n_{i}$ referring to the $\mathscr{N}$ distinct metastable configurations of the entire system. The probabilities satisfy the rate (master) equation $\dot{n}_{i}=\sum_{j=1}^{\mathscr{r}} R_{i j} n_{j}$ with $\sum_{i=1}^{1} n_{i}=1$. The rates $R_{i j}$ of thermally activated transitions from the $i$ th to the $j$ th metastable configuration are of the Arrhenius form $R_{i j} \sim f_{0} \mathrm{e}^{-Q / T}$, where $f_{0}$ is the prefactor. $T$ is temperature $\left(k_{\mathrm{B}}=1\right)$ and $Q$ is the corresponding barrier height.

For $N$ uniaxial particles $\mathscr{N} \leq 2^{N}$ and for practical reasons we limit ourselves to regular arrays of identical particles and to small $N$. We assume coherent rotation and place all particles in parallel with the applied field $\boldsymbol{H}$. The energy of the $\alpha$ th particle becomes

$E_{\alpha}=K V \sin ^{2} \theta_{\alpha}-M_{\mathrm{s}} H V \cos \theta_{\alpha}$,

where $K$ is the anisotropy constant, $V$ the activation volume, $M_{\mathrm{s}}$ the saturation magnetization and the angle $\theta_{\alpha}$ is spanned by the particle's magnetization vector $\boldsymbol{M}_{\alpha}$ and the applied field $\boldsymbol{h}$. The nucleation field $H_{n}=2 K / M_{\mathrm{s}}$ and the magnetostatic coupling energy $[1,2]$ between any two particles is

$E_{\alpha \beta} / V^{2}=r_{\alpha \beta}^{-3}\left(\boldsymbol{M}_{\alpha} \cdot \boldsymbol{M}_{\beta}\right)-3 r_{\alpha \beta}^{-5}\left(\boldsymbol{M}_{\alpha} \cdot \boldsymbol{r}_{\alpha \beta}\right)\left(\boldsymbol{M}_{\beta} \cdot \boldsymbol{r}_{\alpha \beta}\right)$,

$\alpha \neq \beta$, where $r_{\alpha \beta}$ is the radius vector joining the two particles and $r_{\alpha \beta}=\left|r_{\alpha \beta}\right|$. The matrix $R$ of Eq. (1) may be found if all local minima and saddle points ofthe total

" Corresponding author. Email: iklik@phys.sinica.edu.tw; fax: $+886-2-783-4187$. energy $E_{\mathrm{tot}}=\sum_{\alpha}\left(E_{\alpha}+\frac{1}{2} \sum_{\beta \neq \alpha} E_{\alpha \beta}\right)$ are known. This extremal problem has been solved for a pair of identical particles $[2,3]$, but in general the extrema can only be found perturbatively in the limit of a small coupling strength [1]; in this case only single particle reversals are allowed while simultaneous reversals of two or more particles are excluded [1].

The matrix $R$ is derived here, in the weak coupling limit, for a planar particle array deposited on a hexagonal lattice whose properties we compare with previously obtained results [1] for square and diamond planar lattices. The lattices may be stretched by a factor $\xi$ in the easy axes (applied field) direction; the magnetic moment density decreases (increases) on stretching (compression). For an unstretched lattice we define $[1-3]$ the dimensionless coupling constant $\rho=M_{\mathrm{s}}^{2} V /\left(2 \mathrm{Ka}^{3}\right)$, where $a$ is the nearest neighbor distance and, for simplicity, we employ a periodicity assumption [1] under which the entire plane is covered by identical copies of a given metastable configuration of an elementary cell which we choose to be a hexagon with $N=6$ particles. In the low coupling limit each particle experiences a local field which contributes an additional factor [ 1$]$ to the energy (1):

$$
\bar{E}_{\alpha \alpha} /(2 K V \rho)=2 \cos \theta_{\alpha} \sum_{\beta=1}^{6} A_{\alpha \beta} \tilde{p}_{\beta}-3 \sin \theta_{\alpha}\left|\sum_{\beta=1}^{6} B_{\alpha \beta} \tilde{p}_{\beta}\right| .
$$

There exist then $\mathscr{N}=36$ distinct metastable configurations defined by the number sets $\tilde{p}_{\beta}=\cos \theta_{\beta}= \pm 1$; with each configuration we also associate the reduced magnetic moment $\mu_{i}=M_{\mathrm{s}}^{-1} \sum_{\alpha=1}^{6} M_{\alpha}= \pm 6, \pm 4, \pm 2$ and 0 . The small $\rho$ restriction to single particle reversals requires that 


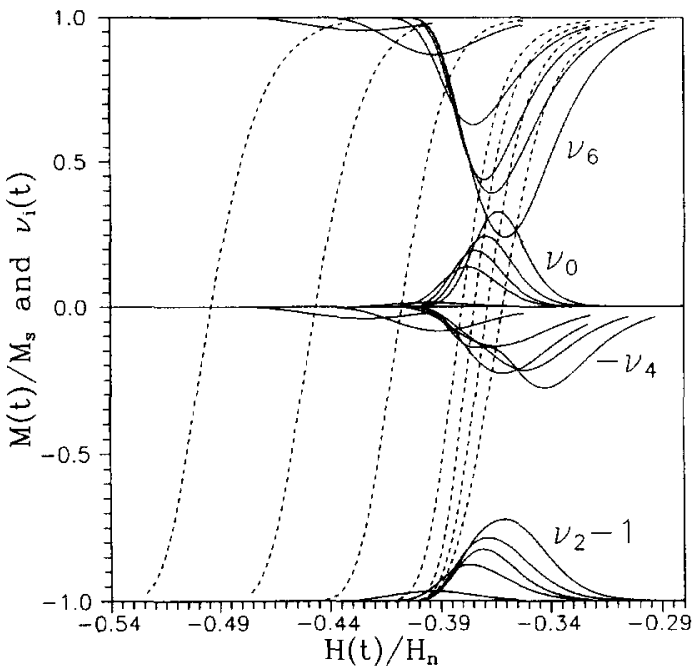

Fig. 1. Sample hysteresis curves for a hexagonal lattice of particles. Shown are the nonequilibrium magnetization $M(t)$ (dashed lines) and the occupation probabilities $\nu_{\mu}(t)$ (solid lines) for stretching factor $\xi=0.5$ (left-most curves with the highest coercivity), $0.58,0.66,0.75,1,1.25,1.5$ and 1.75 (right-most curves). For small $\xi$ there is $\nu_{6}(t) \approx 1$. Coupling strength $\rho=0.015$, $K V / T=42, f_{0}=\mathrm{e}^{25} \mathrm{~Hz}$ and the field sweep rate is $10 \mathrm{~Hz}$.

$\Delta \mu= \pm 2$ in any transition $i \rightarrow j$. The symmetric $6 \times 6$ matrices $A$ and $B$ have here additional symmetries:

$$
\begin{aligned}
A_{\alpha \beta}= & A_{11} \delta_{\alpha, \beta}+A_{12}\left(\delta_{\gamma, 3}+\delta_{\gamma, 6}+\delta_{\gamma, 9}\right) \\
& +A_{13}\left(\bar{\delta}_{\gamma, 4}+\bar{\delta}_{\gamma, 6}\right)+A_{14}\left(\delta_{\gamma, 5}+\delta_{\gamma, 11}\right),
\end{aligned}
$$

$B_{\alpha \beta}=B_{12}\left(\delta_{\gamma, 3}+\delta_{\gamma, 9}-\delta_{\gamma, 7}\right)$ and 0 otherwise, $\gamma=\alpha+\beta$ and $\bar{\delta}_{\gamma, \epsilon}=\delta_{\gamma, \epsilon}\left(1-\delta_{\alpha, \beta}\right)$. The five independent matrix elements may be expressed in terms of the double sums [4]

$$
S(x, y)=\sum_{i, j=-\infty}^{\infty}\left[(x+3 i)^{2}-2 \vec{\xi}^{2}(y+6 j)^{2}\right] / r^{5 / 2} \text {, }
$$

$$
\bar{S}(x, y)=2 \bar{\xi} \sum_{i, j=-x}^{x}(x+3 i)(y+6 j) / r^{5 / 2}
$$

where $\bar{\xi}=3^{1 / 2} \xi / 2$ and $r^{2}=(x+3 i)^{2}+\bar{\xi}^{2}(y+6 j)^{2} \neq 0$ : $A_{11}=S(0,0)+S(3 / 2,3), A_{12}=S(1 / 2,-1)+S(2,2), A_{13}$ $=S(3 / 2,-1)+S(3,2)$ and $A_{14}=S(2,0)+S(1 / 2,3)$ while $B_{12}=\bar{S}(1 / 2,-1)+\bar{S}(2,2)$ in analogy to $A_{12}$.

The immediate magnetization of the elementary cell (and of the entire array) is $M(t)=6^{-1} \sum_{i=1}^{36} \mu_{i} n_{i}(t)$ and Fig. 1 brings, for $\xi \in\langle 0.5,2\rangle$, sample hysteresis curves together with the probabilities $\nu_{\mu}(t)=\left.\sum_{i=1}^{36} n_{i}(t)\right|_{\mu_{i}= \pm \mu}$ that the reduced magnetic moment of the elementary cell hasthe absolute value $\mu=6,4,2$ or 0 . Note that for an isolated particle $\nu_{1} \equiv 1$ at all times. The geometry of the compressed, $\xi=0.5$, lattice is found to favor fully magnetized configurations stabilized by ferromagnetic coupling and Fig. 1 shows that $\nu_{6} \approx 1$ at all times while the occurrence probability of the intermediate partially or fully demagnetized configurations is negligible. The compressed system thus effectively has only two levels, 'all up' and 'all down', and may well be described $[1,3]$ by the customary two level mean field theory [5]. The influence of ferromagnetic coupling in the easy axes direction wanes with increasing $\xi$ where also demagnetized states, stabilized by antiferromagnetic coupling, occur with nonzero probabilities $v_{\mu}, \mu<6$. The stability of the 'all up' state and, consequently, also the coercivity then decrease.

The strong ferromagnetic ordering in the compressed (small $\xi$ ) lattice is easily visualized if one represents the lattice as a series of straight particle chains deposited parallel to the easy axes direction. The interparticle spacing within a chain is $2 \bar{\xi} a$ while the distance between the chains is $a / 2$. The compressed lattice is dominated by ferromagnetic ordering within the chain and this leads to enhanced coercivity (Figs. 1 and 2 ) and switching field distribution (SFD) [3] at coercivity, $\chi_{\mathrm{c}}=\mathrm{d} M /\left.\mathrm{d} H\right|_{H=H_{\mathrm{c}}}$. In Fig. 2 we compare the function $\chi_{c}(\xi)$ for a hexagonal lattice together with our previous results [1] for square and diamond lattices $\left(\mathscr{N}=7\right.$ and 9 respectively and $\left.B_{\alpha \beta} \equiv 0\right)$. Surprisingly, however, in contrast to the coercivity $H_{c}(\xi)$ the SFD function $\chi_{\mathrm{c}}(\xi)$ is not monotonic and Fig. 2 shows that the transition from the two level to the multi-

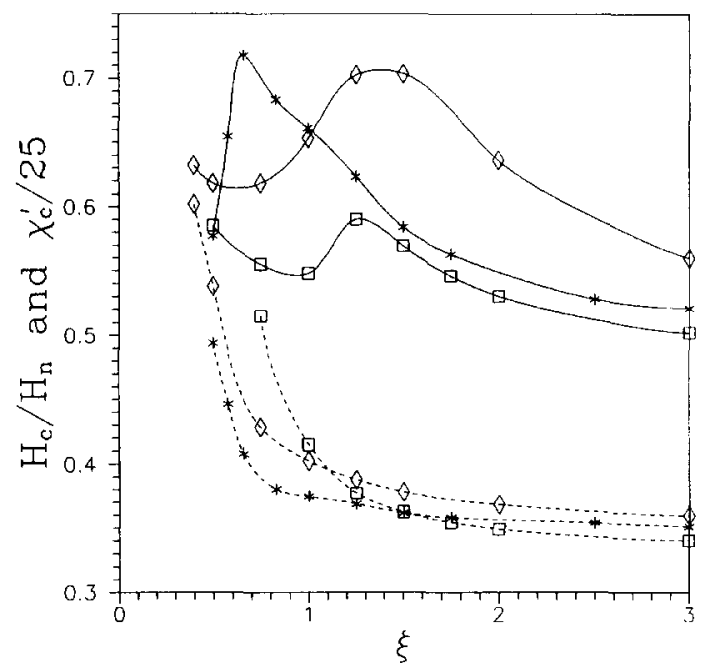

Fig. 2. The coercivity $H_{\mathrm{c}}$ (dashed lines) and the reduced SFD function (solid lines) $\chi_{\mathrm{c}}^{\prime}=\left(H_{\mathrm{n}} / M_{\mathrm{s}}\right) \chi_{\mathrm{c}}$ versus the stretching factor $\xi$ for hexagonal $(*)$, square $(\square)$ and diamond $(\diamond)$ lattices. The local extrema of $\chi_{\mathfrak{c}}$ accompany the transition between two level and multilevel switching regimes (compare Fig. 1). Small $\xi$ data for the hexagonal lattice could not be obtained with sufficient accuracy. Parameters are the same as in Fig. 1, the square and diamond lattice data are taken from Ref. [1]. 
level switching regimes is accompanied by a local maximum; the initial dc susceptibility reaches here its peak value [6]. A similar behavior was also predicted [7] and observed [8] for arrays of noninteracting particles on transitions from the low temperature (irreversible) to the high temperature (reversible) switching regimes. We therefore conclude that a transition between two switching regimes is accompanied by a local decrease of stability against small perturbations.

\section{Acknowledgements}

This research was supported by the National Science Council of the Republic of China under grant No. NSC8421 12-M002-17.

\section{References}

[1] I. Klik and C.R. Chang, Phys. Rev. B 52 (1995) 3540.

[2] W. Chen, S. Zhang and H.N. Bertram, J. Appl. Phys. 71 (1992) 5579.

[3] I. Klik, J.S. Yang and C.R. Chang, J. Appl. Phys. 76 (1994) 6493.

[4] J. Lam. J. Appl. Phys. 72 (1992) 5792

[5] D.K. Lotti, R.M. White and E. Dan Dahiberg, Phys. Rev. Lett. 67 (1991) 362.

[6] I. Klik and C.R. Chang. IEEE Trans. Magn. 31 (1995) 3811.

[7] I. Klik. C.R. Chang and H.L. Huang, Phys. Rev. B 47 (1993) 8605.

[8] J.C. Lin, I. Klik. C.J. Chen and C.R. Chang. J. Appl. Phys. 75 (1994) 5897. 